\title{
Aging, autonomic function, and the perception of angina
}

V Umachandran, K Ranjadayalan, G Ambepityia, B Marchant, P G Kopelman, A D Timmis

\begin{abstract}
Objective-To determine the effects of age and autonomic function on the perception of angina.

Design-Prospective evaluation of the relations between anginal perceptual threshold, autonomic function, and systolic blood pressure in patients with symptomatic coronary artery disease. Statistical analysis was by nonparametric techniques.

Setting-Cardiology departments of a district general hospital and a postgraduate teaching centre.

Subjects-82 non-diabetic men with typical exertional angina and coronary artery disease confirmed by arteriography $(n=64)$ or a history of $Q$ wave infarction $(n=18)$.

Main outcome measures-Age, anginal perceptual threshold, autonomic function, and blood pressure. Anginal perceptual threshold was defined as the time from onset of $0.1 \mathrm{mV}$ ST depression to the onset of angina during treadmill stress testing. Autonomic function was measured as the ratio of peak heart rate during the Valsalva manoeuvre to the minimum rate after release.
\end{abstract}

Results-Anginal perceptual threshold showed a weak but significant correlation with age, with older patients tending to have a longer interval between the onset of ST depression and the onset of angina. Comparison of patients in the upper and lower quartile age ranges showed a difference of 50 seconds between median threshold measurements. Blood pressure and heart rate responses to the Valsalva manoeuvre also correlated with age, but neither variable correlated with the anginal perceptual threshold.

Conclusions-In non-diabetic men with coronary artery disease the perception of angina tends to deteriorate with advancing age. The mechanism is unclear but is not attributable solely to alterations in blood pressure or autonomic function.

In patients with coronary artery disease angina usually occurs in response to exercise induced myocardial ischaemia. However, there is often a latent interval after the provocation of myocardial ischaemia before chest pain develops. We have called this latent interval the anginal perceptual threshold and have defined it as the time from onset of $0.1 \mathrm{mV} \mathrm{ST} \mathrm{depression} \mathrm{to} \mathrm{the} \mathrm{onset} \mathrm{of} \mathrm{angina}$ during treadmill stress testing. Because the anginal perceptual threshold is a continuous variable it provides a useful clinical measure of the perception of angina and may be useful for investigating the mechanisms responsible for silent ischaemia.

Preliminary work has shown that the anginal perceptual threshold is prolonged in diabetes, particularly in patients with autonomic neuropathy, ${ }^{1}$ which means that damage to the sensory innervation of the heart may account for the propensity to silent ischaemia in these patients. ${ }^{2-4}$ It is not known, however, whether neuropathic mechanisms are active in the silent ischaemia seen in non-diabetic patients with coronary artery disease. ${ }^{56}$ If neuropathic mechanisms are important in the non-diabetic population, alterations in the perception of angina would be expected to be more common in the elderly because of the tendency for autonomic ${ }^{78}$ and sensory ${ }^{9}{ }^{10}$ function to deteriorate with increasing age. The Framingham study showed that asymptomatic myocardial infarction is indeed more common in elderly people ${ }^{11}$ but information about the effects of aging on the perception of angina is scarce. This information is potentially important not only because the proportion of elderly people is increasing but also because of the insights it might provide into the mechanisms of silent ischaemia. We have measured the anginal perceptual threshold in a group of non-diabetic men with exertional angina to determine the effects of age and autonomic function on the perception of angina.

\section{Patients and methods}

PATIENTS

The 82 patients included in this study were non-diabetic men (median age 63 years (range 35-82)). All had typical exertional angina and coronary artery disease confirmed by arteriography $(n=64)$ or a history of $Q$ wave infarction $(n=18)$. None had clinical evidence of autonomic or peripheral neuropathy. Patients were required to be in sinus rhythm and were excluded if they had unstable angina, myocardial infarction within the previous three months, or electrocardiographic abnormalities preventing adequate interpretation of the exercise stress test (left bundle branch block, digoxin induced changes in the ST segment or $T$ wave). We 
also excluded patients with intermittent claudication or musculoskeletal disorders severe enough to interfere with exercise testing. All patients gave written informed consent for participation in this study which had been approved by the Newham Health District Ethical Committee.

TREADMILL STRESS TESTING

The stress tests were all performed by one of three experienced doctors (KR, VU, or GA) and in none of our patients did we rely on clinical observations from paramedical staff. Aspirin and antianginal drugs, except for sublingual glyceryl trinitrate, were stopped at least 12 hours beforehand. Symptom limit exercise testing was carried out on a motor driven treadmill by the Bruce protocol. ${ }^{12}$ Patients were exercised for three minute stages at progressively increasing workloads. A 12 lead electrocardiogram was recorded before exercise, at the end of each three minute stage, at peak exercise, and every minute during recovery. Heart rate and blood pressure were recorded at the same intervals. In addition, the three leads previously identified as showing the earliest ST depression were recorded continuously throughout exercise on the electrocardiographic print out in order to identify the time to onset of $0.1 \mathrm{mV}$ ST depression measured $80 \mathrm{~ms}$ after the J point. The patients were asked to report the onset of angina and to ensure that the time was accurately identified the doctor inquired about symptoms throughout the test. The anginal perceptual threshold was calculated as the time from the onset of $0.1 \mathrm{mV} \mathrm{ST}$ depression to the onset of angina. Three patients who did not experience chest pain during the stress test despite diagnostic ST segment depression were excluded from the analysis of the anginal perceptual threshold.

AUTONOMIC FUNCTION TESTING

This was performed immediately before the treadmill stress test. In every patient heart rate was monitored on the electrocardiogram during and after the Valsalva manoeuvre (40 $\mathrm{mm} \mathrm{Hg}$ measured against a mercury column). We used the ratio of the peak heart rate during the Valsalva manoeuvre to the minimum rate after release as an index of autonomic function. ${ }^{13}$

Results of treadmill stress tests (median (interquartile range))

\begin{tabular}{lc}
\hline Variable & Result \\
\hline Heart rate (beats/min) & $77(67-84)$ \\
$\quad$ Rest & $130(116-140)$ \\
Peak & $140(120-150)$ \\
Systolic blood pressure (mm Hg) & $160(140-175)$ \\
$\quad$ Rest & $20.6(17 \cdot 0-23 \cdot 3)$ \\
$\quad$ Peak & $0.6(0 \cdot 4-0 \cdot 8)$ \\
$\begin{array}{l}\text { Peak rate-pressure product } \\
\left.\text { (mm Hg beats/min } \times 10^{-3}\right)\end{array}$ & $193(120-282)$ \\
$\begin{array}{l}\text { Sum of ST depression } \\
\text { at peak exercise }(\mathrm{mV})\end{array}$ & $228(147-325)$ \\
Time to onset of $0.1 \mathrm{mV}$ & $40(10-64)$ \\
$\quad$ ST depression $(\mathrm{s})$ &
\end{tabular}

\section{STATIS'TICAL ANALYSIS}

All averaged results were expressed as medians with the interquartile range in parentheses. Non-parametric tests were used for all data comparisons. Correlations between age, blood pressure, autonomic function, and the anginal perceptual threshold were calculated by Spearman's rank method. The MannWhitney test was used to compare differences between patients in the upper and lower age quartiles. A two tailed $p$ value of $<0.05$ was regarded as statistically significant.

\section{Results}

ANGINAL PERCEPTUAL THRESHOLD (TABLE)

Treadmill exercise produced physiological increases in blood pressure and heart rate. Every patient developed at least $0.1 \mathrm{mV}$ of planar or down sloping ST segment depression after $193(120-282) \mathrm{s}$ of exercise with the onset of angina in all but three cases after 228 (147-325) s. In the 79 patients who experienced angina, the anginal perceptual threshold was 40 $(10-64) \mathrm{s}$. The age of these patients ranged from 35 to 82 years and showed a weak but significant correlation with the anginal perceptual threshold $(r=0.33, p<0.005)$, with older patients tending to have a longer latent interval between the onset of ST depression and the onset of angina (fig 1). Thus comparison of the anginal perceptual thresholds in the upper ( $\geq 68$ years) and lower ( $\leq 56$ years) quartile age groups confirmed a highly significant difference between the median values $(64(38-98) v 14(-5-51), \mathrm{p}<0.005)$ (fig 2). Neither myocardial ischaemia nor oxygen demand at peak exercise (as judged by the sum of ST depression and the rate-pressure product respectively) correlated with the anginal perceptual threshold.

AUTONOMIC FUNCTION AND BLOOD PRESSURE Autonomic function, as reflected by heart rate responses to the Valsalva manoeuvre, showed the expected age related changes. Thus in older patients the change in heart rate tended to be less acute during the Valsalva manoeuvre with

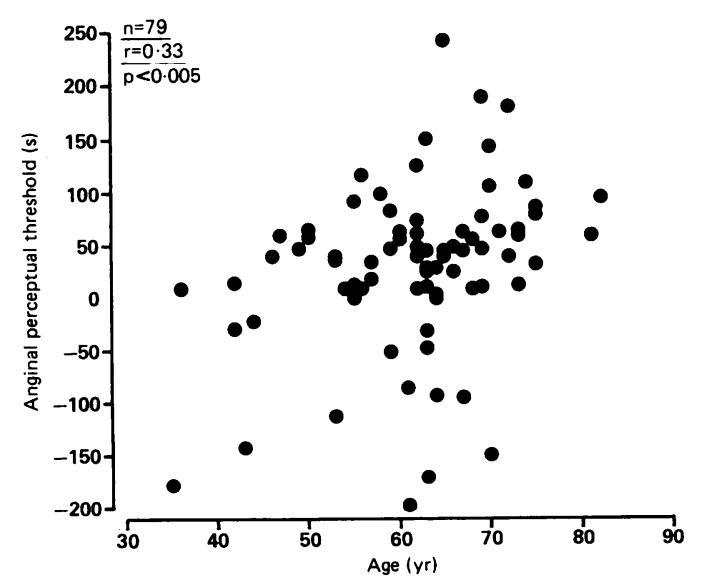

Figure 1 Scattergram (with fitted simple regression) showing relation between age and anginal perceptual threshold. Superimposition of two pairs of data points has reduced by two the number of points visible in the scattergram. 
Figure 2 Comparison of anginal perceptual patients in the upper $1 \geqslant 68$ years) and lower $1 \geqslant 68$ years $)$ and lower
$(\leq 56$ years) quartile age ranges. Each box plot 75 th, and 90 th percentile values. The extreme $20 \%$ below the 10th percentile and $10 \%$ above the 90 th percentile) are shown as open circles. Notches in the box chart represent $95 \%$ confidence bands about the median. threshold measurements in shows the 10th, 25th, 50th of observed values $(10 \%$

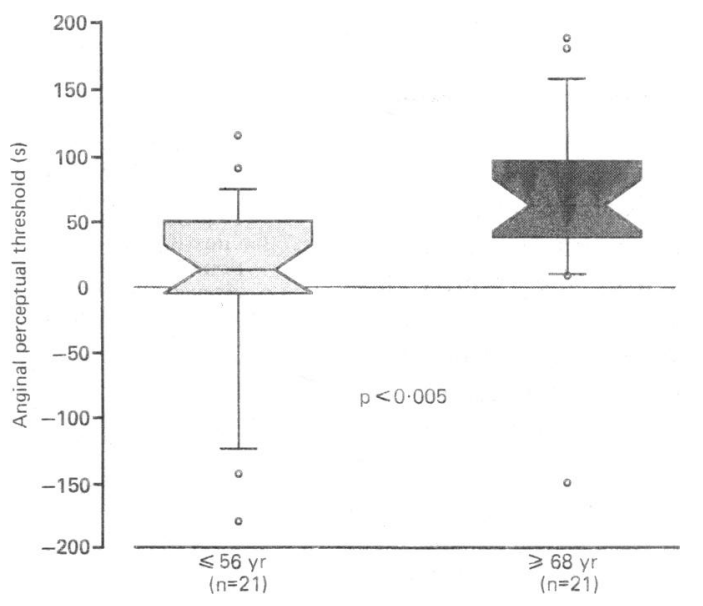

a lower ratio of peak to minimum values (fig 3 ). Systolic blood pressure also showed the expected correlation with age, older patients tending to have higher systolic measurements (fig 3). Neither autonomic function nor blood pressure, however, showed any relation to the anginal perceptual threshold.

\section{Discussion}

This study showed that in non-diabetic men with coronary artery disease the perception of exertional angina shows a weak but significant relation with the age of the patient. With advancing age the latent interval between the onset of regional myocardial ischaemia and the onset of chest pain (the anginal perceptual threshold) becomes longer, such that comparison of patients in the upper and lower quartiles for age range showed a difference of

Figure 3 Scattergrams (with fitted simple regression) showing relations between age, systolic blood pressure, and autonomic function. Superimposition of paired data points (blood pressure 10; Valsalva 1) has caused corresponding reductions in the number of points visible in each scattergram.
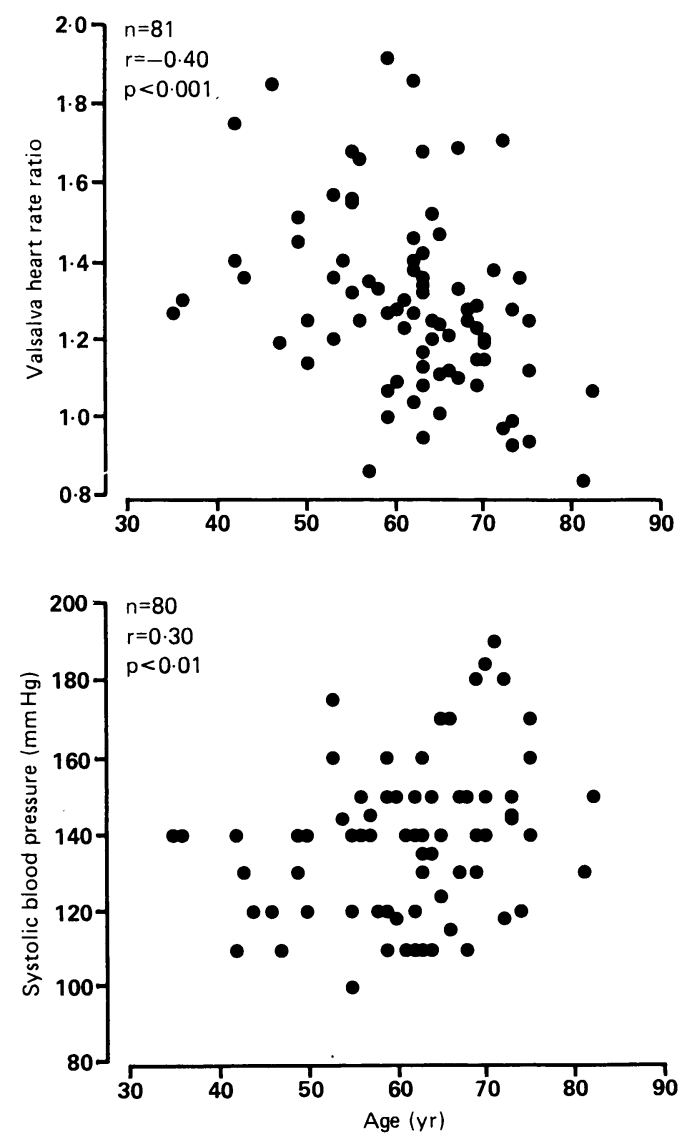

$50 \mathrm{~s}$ between median threshold measurements. Although there were the expected relations between age, blood pressure, and heart rate responses to the Valsalva manoeuvre, only age correlated with the anginal perceptual threshold indicating that changes in the perception of angina in the elderly are not attributable solely to alterations in autonomic function.

The anginal perceptual threshold provides a useful index of the perception of angina in patients wth coronary artery disease. Earlier work showed that the threshold can be considerably prolonged in diabetic patients who often do not experience angina until two minutes or longer after the onset of exertional ST depression. ${ }^{1}$ Prolongation of the anginal perceptual threshold in diabetes is particularly exaggerated in patients with abnormal autonomic function and is commonly associated with a heightened somatic pain threshold, ${ }^{14}$ indicating that the perceptual impairment is generalised and is caused, at least in part, by diabetic neuropathy. ${ }^{4}$ The present study indicates that with advancing age a similar, though less pronounced, hyposensitivity to ischaemic cardiac pain develops in nondiabetic patients. The correlation is highly significant but weak, indicating that other factors apart from age are likely to have an independent effect on the perception of angina. Nevertheless, our data suggest that neuropathic mechanisms are less important than in diabetic patients with coronary artery disease.

Recently, Miller et al reported age related changes in the perception of angina similar to those in the present study. ${ }^{15}$ These changes were independent of the severity of ischaemia, which supports our own findings that myocardial ischaemia and oxygen demand at peak exercise (as judged by the sum of ST depression and the rate-pressure product, respectively) were unrelated to the anginal perceptual threshold. Miller et al suggested that the dysfunctional perception might reflect the tendency for blood pressure to rise with age; this suggestion was based on previous reports of increased pain thresholds in hypertensive patients. ${ }^{16}{ }^{17}$ It accorded with the work of Sheps et al who described a relation between systolic blood pressure and silent ischaemia in patients with angina and identified a potential role for altered baroreceptor function. ${ }^{18}$

The sensory supply to the heart is part of the autonomic innervation and changes in autonomic function and blood pressure certainly provide a plausible explanation for the agerelated increase in the anginal perceptual threshold shown in the present study. However, although our data showed the expected changes in autonomic function and blood pressure in the older age group, neither of these variables was related to the anginal perceptual threshold; this indicated that other mechanisms must contribute to the impairment of perception with increasing age. A generalised hyposensitivity to pain has been seen in the elderly $^{910}$ and it has been suggested that this might be the result of central modulation by endogenous opioid peptides, ${ }^{19}$ though whether this accounts for the prolongation of the 
anginal perceptual threshold with advancing age remains speculative. Indeed, the mechanism of hypoalgesia in the elderly is at present uncertain but is likely to be multifactorial involving functional alterations in the peripheral and central nervous systems.

The increase in the anginal perceptual threshold with advancing age seems less pronounced than in diabetic patients who can develop profound exertional ischaemia before they experience angina. ${ }^{20}$ Nevertheless, any delay in the perception of angina during exertional ischaemia will increase the ischaemic burden in the elderly which may in turn heighten the risk of cardiac arrhythmias and ongoing myofibrillar damage. A similar risk has been ascribed to patients with silent ischaemia and clearly there may be some heterogeneity between silent ischaemia and prolongation of the anginal perceptual threshold. ${ }^{21}$ It is not known, however, whether the tendency for the anginal perceptual threshold to increase in elderly patients with coronary artery disease is associated with an increase in the prevalence of silent ischaemia and more work is necessary to define the relation between these perceptual disorders and to determine their clinical significance.

1 Ambepityia G, Kopelman PG, Ingram D, Swash M, Mills $P G$, Timmis AD. Exertional myocardial ischemia in diabetes: a quantitative analysis of anginal perceptual threshold and the influence of autonomic function. J Am Coll Cardiol 1990;15:72-7.

2 Chiariello M, Indolfi C, Cotecchia MR, Sifola C, Romano M, Condorelli M. Asymptomatic transient ST changes during ambulatory ECG monitoring in diabetic patients. during ambulatory ECG mon

3 Nesto RW, Phillips RT, Kett KG, et al. Angina and exertional myocardial ischemia in diabetic and nondiabetic patients: assessment by exercise thallium scintigraphy. Ann Intern Med 1988;108:170-5.
4 Murray DP, O'Brien T, Mulrooney R, O'Sullivan DJ. Autonomic dysfunction and silent myocardial ischaemia on exercise testing in diabetes mellitus. Diabetic Med 1990;7:580-4.

5 Fox KM. Silent ischaemia: clinical implications in 1988. Br Heart J 1988;60:363-6.

6 Sheps DS, Maixner W, Hinderliter AL. Mechanisms of pain perception in silent myocardial ischemia. Am Heart $J$ 1990;119:983-7.

7 Collins KJ, Exton-Sith AK, James MH, Oliver DJ. Functional changes in autonomic responses with ageing. Age tional changes in au

8 O'Brien IAD, O'Hare $\mathrm{P}$, Corrall RJM. Heart rate variability in healthy subjects: effect of age and the derivation of normal ranges for tests of autonomic function. Br Heart $J$ normal ranges fo

9 Harkins SW, Price DP, Martelli M. Effects of age on pain perception: thermonociception. J Gerentol 1986;41:58-63. 10 Neri M, Agazzani E. Aging and right-left asymmetry in experimental pain measurement. Pain 1984;19:43-8.

11 Kannel WB, Abbott RD. Incidence and prognosis of unrecognized myocardial infarction: an update on the Framingham study. $N$ Engl J Med 1984;311:1144-7.

12 Bruce RA. Exercise testing of patients with coronary heart disease. Ann Clin Res 1971;3:323-32.

13 Ewing DJ, Clarke BF. Diagnosis and management of diabetic autonomic neuropathy. $B M J$ 1982;285:916-9.

14 Umachandran V, Ranjadayalan K, Ambepityia G, Marchant B, Kopelman PG, Timmis AD. The perception of angina in diabetes: relation to somatic pain threshold and autoin diabetes: relation to somatic pain the

15 Miller PF, Sheps DS, Bragdon EE, et al. Aging and pain perception in ischemic disease. Am Heart $f$ 1990;120: percep

16 Dworkin BR, Filewich RJ, Miller NE, Craigmyle N, Pickering TG. Baroreceptor activation reduces reactivity to noxious stimulation: implications for hypertension. Science 1979;205:1299-301

17 Ghione S, Rosa C, Mezzasalma L, Panattoni E. Arterial hypertension is associated with hyperalgesia in humans. Hypertension 1988;12:491-7.

18 Sheps DS, Maixner W, Hinderliter AL, et al. The relationship between systolic blood pressure, ventricular volume and ischemic pain perception in patients with angina pectoris: a potential role for baroreceptors. Isr J Med $S c i$ 1989;25:482-7.

19 Saavendra JM. Naloxone reversible decrease in pain sensitivity in young and adult spontaneously hypertensive rats. Brain Res 1987;209:245-9.

20 Ranjadayalan K, Umachandran V, Ambepityia G, Kopelman PG, Mills PG, Timmis AD. Prolonged anginal perceptual threshold in diabetes: effects on exercise perceptual threshold in diabetes: effects on exercise capacity and my

21 Cohn PF. Silent myocardial ischemia in patients with a defective anginal warning system. Am J Cardiol 1980; 45:697-702. 